\title{
Sellers' hedging incentives at EPA's emission trading auction
}

\author{
Bouwe R. Dijkstra*and Marco Haan
}

January 8, 1999

\begin{abstract}
Cason (1993) argued that the auction the EPA used in order to start the market for sulfur allowances, is not efficient. The set-up of the auction gives both buyers and sellers an incentive to understate their valuation of an allowance. In this paper, we show that the sellers' incentives are even more perverse than Cason suggested. In particular, we show that sellers have an incentive to set their ask price equal to zero, while simultaneously hedging their bets by submitting a positive bid. It is not possible to derive the Nash equilibrium for this set-up. If such an equilibrium exists, sellers either set only a positive ask price, or an ask price equal to zero, and a positive bid as well.
\end{abstract}

\footnotetext{
* Department of Economics, Faculty of Law, University of Groningen, PO Box 716, 9700 AS Groningen, fax: +3150363 7101, email: b.r.dijkstra@ rechten.rug.nl.

Faculty of Economics, Department of Micro-economics, University of Groningen, P.O. Box 800, 9700 AV Groningen, The Netherlands, fax: +31-50-3637337. e-mail: m.a.haan@eco.rug.nl. The authors thank Lambert Schoonbeek for useful comments.
} 


\section{Introduction}

Since Dales (1968), economists have recommended tradeable permits as an efficient instrument of environmental policy. However, the development of this instrument in policy practice has been slow. In the 1970s, emissions trading rules emerged in the US. Because there was no conscious design, the rules were unclear and controversial and required a lot of government intervention (Liroff 1986).

In 1990, the first large-scale, consciously designed emission trading scheme was introduced in the US. It was applied to the $\mathrm{SO}_{2}$ emissions of electric utilities. ${ }^{1}$ In Phase I (1995-2000), a limited number of electric utilities participate in the scheme. In Phase II (from 2000), all electric utilities will participate.

Sulfur allowances can be traded in two different ways. One way is to trade privately between utilities, possibly with the intermediation of a broker. By now, the lion's share of allowances is traded in this way. The other trading option is the annual auction in March, organized by the EPA. This auction was first held in 1993. At the auction, the EPA sells the small part (2.8\%) of the total amount of allowances that is not grandfathered directly to the utilities. The revenues of the auction are distributed among the allowance holders. Electric utilities and other interested parties can not only bid at the auction, but also offer allowances for sale.

The way in which the auction is conducted is unique. ${ }^{2}$ The bids are ranked from high to low. The allowances from the EPA are sold to the highest bidders. The other suppliers are ranked according to their ask price, from low to high. The lowest asker is matched to the highest remaining bidder, etc. as long as the ask price is below the bid price. A successful bidder pays his bid price to the seller to whom he is matched.

This was the first time that this auction design was implemented. Also, it had not been analyzed before. Cason (1993) is the only paper which provides an analysis of the auction. ${ }^{3} \mathrm{He}$ claims that this auction is inefficient, in that the market-clearing prices are too low and not all gains from trade are exhausted. In a standard uniform price auction, all trades take place at the market-clearing price, given all bid and ask prices submitted. In such an auction, buyers have an incentive to bid slightly lower than their valuation, whereas sellers have an incentive to ask a price that is slightly

1 Ellerman et al. (1997) provide a comprehensive report of the whole program. Schmalensee et al. (1998) summarize the report. Joskow et al. (1998) discuss the allowance market. Stavins (1998) discusses the positive and normative lessons from the program.

2 This way of conducting the auction is not explicity laid down in the 1990 Clean Air Act Amendments (CAAA). Rather, it is based upon EPA's interpretation of the CAAA (Cason, 1993).

3 In the remainder of this paper, we will refer to Cason (1993) simply as Cason. 
higher than their valuation (see for example Vickrey, 1961, or McAfee and McMillan, 1987). This results in an efficient market. In the EPA auction, however, Cason shows that a seller has an incentive to offer units at prices below her costs. Given that she will sell, the lower her ask price, the higher the bid to which she will be matched, and therefore the higher her expected revenue. Given that bidders still have an incentive to bid below their valuations, this results in biased price signals which may reduce the efficiency of the market.

However, as we will show in this paper, Cason's analysis is incomplete. He only looks at the ask price that allowance sellers will set. We will show that it may be more profitable for a seller to simultaneously set an ask price equal to zero and submit a bid. As a result, the seller's incentives in this auction are even more perverse than Cason suggested.

We now know that the auction did not live up to its expectations. It was seen as a "market starter". The first auction was held in 1993, whereas the program would start in 1995. The auction was supposed to give an indication of the allowance price to be used as a guideline for private trades. ${ }^{4}$ Furthermore, once the program was under way, if electric utilities found it difficult to buy or sell at the private market, they could always resort to the auction.

\section{INSERT TABLE 1 ABOUT HERE}

As Table 1 shows, the relative importance of the auction has declined through the years, as has private supply at the auction. In 1993 and 1994, the quantity of private supply was about the same as the quantity offered by the EPA. However, only up to two per cent of privately supplied allowances was sold. In 1995, private supply declined drastically, whereas the sales remained at the same level. In 1996, none of the private supply was sold. From 1997 on, there has not been any private supply at the auction.

Despite (or perhaps due to) the disappointing performance of the auction, a flourishing and efficient private market did develop. We see in Table 1 that the ratio of privately traded allowances to allowances sold at the auction has risen from 5 in 1994 to 28 in 1997. Therefore, Cason's fear ${ }^{5}$ seems to have proven unfounded.

4 In fact, the market-clearing price of the first EPA auction in 1993 was below the average price of the scarce private transactions around that time. From 1994 on, the prices on both markets were very close (See Ellerman et al., 1997, or Schmalensee et al., 1998).

5 "If this highly visible market experiment fails because Congress designed the trading mechanism 
Why should we then want to analyze the EPA auction, now that it has proven to be a less relevant trading option, at least for private supply? First, there is the intellectual challenge of finding an equilibrium to this auction. Second, with a better understanding of how the auction works we may be able to explain why it failed to be relevant. An auction with a different design might have been a more attractive trading option and might have given a more reliable price signal to the private market. This is also relevant for future auctions of tradeable emission permits or other items. Third, we show that market participants can make money in the EPA auctions by using our approach rather than the equilibrium strategy derived by Cason. ${ }^{6}$

The remainder of this paper is organized as follows. In Section 2, we introduce the formal description of the players and the rules of the auction. We also discuss the equilibrium derived by Cason. In this equilibrium, sellers only set an ask price. In Section 3, we show that when all other sellers follow Cason's strategy, any one seller can achieve a higher payoff by submitting an ask and a bid price, rather than only an ask price. In section 4 we derive some characteristics of the appropriate equilibrium in this auction. In particular, we show that in any equilibrium, sellers either submit an ask price equal to zero, plus a positive bid, or they only submit a positive ask price, and no bid. In section 5 we sketch the derivation of the appropriate equilibrium, and show that (at least, for us) it is impossible to solve. Section 6 concludes the paper.

\section{The Model}

In this section, we introduce the formal model to analyze the EPA auction, and also present the solution Cason has given for the equilibrium strategies of the sellers. Cason makes the following simplifying assumptions. First, assume that sellers offer only one unit for sale in the auction. Second, the auction is the only way to trade allowances. Third, we do not take the units offered by the EPA into account. Fourth, we abstract from strategic buyers by assuming that bids are drawn from some probability distribution $\Phi(\cdot)$ with density whose support is the interval $[\underline{b}, \bar{b}]$. Fifth, both buyers and sellers are risk neutral.

Suppose we have $Q$ bids. Each of $N \geq Q$ sellers submits an ask price to sell a single unit. Random bid prices are unknown when sellers enter their asks. Rank the

poorly, the negative impact on future regulatory policy may extend well beyond federal acid rain legislation." (Cason, 1993, p. 178).

6 Cason also conducted a series of experiments to test whether subjects behave in the way described by his model (Cason 1995, Cason and Plott 1996). In these experiments, however, sellers did not have the possibility to submit a bid. 
$Q$ bids in decreasing order: $b_{1} \geq b_{2} \geq \ldots \geq b_{Q}$, and the $N$ asks in increasing order: $a_{1} \leq a_{2} \leq \ldots a_{N}$. The seller asking $a_{j}$ is matched with bid $b_{j}$ and receives a price equal to $b_{j}$ only if $b_{j} \geq a_{j}$. Let $c_{i}$ denote the emission compliance cost of seller $i$, $i=1, \ldots, N$. Each $c_{i}$ is drawn from a known probability distribution function $H(\cdot)$ with density $h(\cdot)$, whose support is $[\underline{c}, \bar{c}]$. If seller $i$ sells the unit matched with $b_{j}$ she gains $\left\{b_{j}-c_{i}\right\}$. If not, she earns zero. A strictly positive equilibrium ask function $a_{i}=a\left(c_{i}\right)$ can be derived as a symmetric Bayesian Nash equilibrium, provided that $\underline{c}$ is high enough, as is assumed by Cason.

When determining her optimal ask price $a_{i}$, the seller must trade off two effects. On the one hand, a decrease in $a_{i}$ results in a higher expected bid price to which she will be matched and therefore in a higher expected payoff. However, this is only the case when the initial ask price was already low enough for a sale to take place. It can also happen that the initial ask price is too high, and there will not be a sale. Then, when the seller reduces her ask price, she may succeed in selling her allowance, but will make a loss when the bid price to which she is matched is below her own $\operatorname{cost} c_{i}$. For a uniform distribution of bids and sellers' costs, Cason shows that the equilibrium ask function has $a\left(c_{i}\right)<c_{i}$. Hence, the optimal ask is below the seller's cost $c_{i}$, and there is a possibility that, in equilibrium, a seller makes a loss.

\section{Why This Is Not An Equilibrium}

In this section, we prove that the equilibrium described by Cason, in which every seller $i$ sets a strictly positive ask $a_{i}$, is no longer a Nash equilibrium if we also allow sellers to submit a bid price. Indeed, we will show that, in that case, any situation in which all participating sellers set a strictly positive ask price, does not qualify as a Nash equilibrium. Every seller can improve by submitting an ask price zero instead, and submitting a positive bid as well. One way to break the equilibrium is the following

Theorem 3.1 Any situation in which all participating sellers set a strictly positive ask price, cannot be a Nash equilibrium, since every seller can strictly improve by simultaneously submitting an ask price equal to zero, and a bid price equal to her $\operatorname{cost} c_{i}$.

Proof. Consider the situation described in the theorem, in which all submitted asks are strictly positive. For ease of exposition, we will refer to the strategy used in this case by seller $i$ as the strictly positive strategy. The agents in the auction 
submit $Q$ bids, given, in descending order, by $b_{1}>b_{2}>\ldots>b_{Q} \cdot{ }^{7,8}$ We sort all asks in ascending order, and define $r$ as the rank of some seller $i$ 's ask. Thus $0<a_{1}<a_{2}<\ldots<a_{r-1}<a_{r}<a_{r+1} \ldots$, where $a_{r}$ is the ask submitted by seller $i$. We will show that seller $i$ is better off using the alternative strategy described in theorem 3.1, rather than the strictly positive strategy.

Define the marginal bidder $m$ as the bidder with the lowest successful bid:

$$
m=\max _{j}\left\{j: b_{j}>a_{j}\right\} .
$$

If seller $i$ sells her allowance, she will receive an amount $b_{r}$, which is the bid matched to her ask $a_{r}$. Since she values the allowance at $c_{i}$, her payoff then equals $b_{r}-c_{i}$. Hence, seller $i$ 's net payoff $\pi_{i}$ from the strictly positive strategy equals

$$
\pi_{i}=\left\{\begin{array}{cc}
b_{r}-c_{i} & \text { if } r \leq m \\
0 & \text { if } r>m
\end{array}\right.
$$

Now consider the alternative described in the theorem. First suppose seller $i$ only submits an ask $a_{i}=0$, rather than the $a_{i}>0$ we had above. Given the strategies of the other sellers, she now receives the highest bid with certainty. Her payoff thus equals $b_{1}-c_{i}$. This expression is positive if and only if $b_{1}>c_{i}$. But seller $i$ can avoid making a loss by also submitting a bid $B_{i}=c_{i}$. In that case, should we have $b_{1}<c_{i}$, the highest bid will be her own, and the seller effectively buys her own allowance, thus avoiding a loss. In other cases, the seller is indifferent between whether or not $B_{i}$ is successful, since $B_{i}$ is exactly equal to her valuation of an allowance. Therefore, using this strategy yields a payoff

$$
\pi_{i}=\left\{\begin{array}{cc}
b_{1}-c_{i} & \text { if } b_{1}>c_{i} \\
0 & \text { if } b_{1} \leq c_{i}
\end{array}\right.
$$

To compare the payoffs in (2) and (3), we have three cases to consider.

1. In case it turns out that both $r \leq m$ and $b_{r}<c_{i}$, using the strictly positive strategy yields a negative payoff, while the payoff of using the alternative strategy is either positive (if also $b_{1}>c_{i}$ ), or equal to zero (if this is not the case).

7 Since both asks and bids are drawn from continuous distributions, there is a probability of 0 that any two are equal. Therefore, we can use strict inequalities instead of weak inequalities.

8 Note that this is only for ease of exposition; seller $i$ does not know the values of the other asks and bids. The only thing she possibly knows are the equilibrium strategies which map the valuations of the other participants into asks or bids. 
2. If it turns out that $r \leq m$ and $b_{r}>c_{i}$, the strictly positive strategy yields $b_{r}-c_{i}$. The alternative strategy now yields $b_{1}-c_{i}$, which is equal if $r=1$, and higher otherwise.

3. If it turns out that $r>m$, the strictly positive strategy yields 0 , while the alternative strategy yields a strictly positive payoff if $b_{1}>c_{i}$, and zero otherwise.

Thus, in each possible case the seller is at least as well off using the alternative strategy, than she is using the strictly positive strategy. Moreover, in the first case, she is strictly better off using the alternative strategy. Therefore, for seller $i$, the alternative strategy strictly dominates the strictly positive strategy, which implies that the latter is not a Nash equilibrium.

From theorem 3.1, we immediately have

Corollary 3.1 The equilibrium described by Cason (1993) is not a Nash equilibrium when sellers can also submit a bid price.

\section{Properties of the Appropriate Nash Equilibrium}

The result in the previous section suggests that, to find an equilibrium in this auction, we have to use an extended strategy space for the sellers. Rather than just submitting an ask price $a_{i}$, we now consider the case in which a seller submits both an ask $a_{i}$ and a bid price $B_{i}$. In this section we will derive some properties of the appropriate Nash equilibrium in the auction considered. We will show that, in any pure strategy equilibrium, any seller will either set an ask equal to zero and a strictly positive bid price, or only a strictly positive ask price. This result is used in the next section to sketch the derivation of the appropriate Nash equilibrium.

We assume, as in Cason, that every buyer and seller only needs one allowance. Therefore, for each agent, the valuation of a second allowance equals zero. Also, assume that ties are broken at random. Note that the payoff to seller $i$ now consists of two parts: the payoff of (possibly) buying an allowance, and the payoff of (possibly) selling one. Since we no longer restrict attention to the equilibrium strategies described by Cason, we now explicitly have to take the possibility into account that other sellers set an ask price 0 , and/or a positive bid price as well. Suppose that, among the $N-1$ other sellers, $N_{b}$ submit a bid price, and $N_{a}$ submit an ask price. Among the other sellers who are submitting an ask price, $z$ set ask price zero. The other market participants thus submit a total of $Q+N_{b}$ bids, and a total of $N_{a}$ asks. Order all bids 
of the participants other than $i$ such that $b_{1}>b_{2}>\ldots>b_{Q+N_{b}}$. Order all asks, including that of seller $i$, such that $0=a_{1}=a_{2}=\ldots=a_{z} \leq a_{z+1}<\ldots<a_{N_{a}} \cdot{ }^{9}$ Define the marginal bidder $m$ as the one with the lowest successful bid, again not taking the bid by seller $i$ into account, as in (1). If seller $i$ submits a bid, we denote it by $B_{i}$. Define the series $\beta_{1}>\beta_{2} \ldots>\beta_{Q+N_{b}+1}$ which orders all bids when $B_{i}$ is also taken into account. For this new situation, define $M$ as the marginal bidder:

$$
M=\max _{j}\left\{j: \beta_{j}>a_{j}\right\} .
$$

We first prove the following lemma

Lemma 4.1 When $B_{i}<a_{i}$ and $a_{i}$ is successful, then $B_{i}$ cannot be successful.

Proof. When $a_{i}$ is successful, we have $a_{i} \leq a_{M}$. By definition of the marginal bidder, we have $a_{M}<\beta_{M}$. Thus $B_{i}<a_{i} \leq a_{M}<\beta_{M}$. But $B_{i}<\beta_{M}$ implies that $B_{i}$ is not successful.

Also,

Lemma 4.2 When $a_{i} \leq B_{i}$, and $B_{i}$ is not successful, then $a_{i}$ is successful.

Proof. When $B_{i}$ is not successful, we necessarily have $B_{i} \leq \beta_{M+1}$. Since, by definition, $a_{M+1}>\beta_{M+1}$, we have $a_{i} \leq B_{i} \leq \beta_{M+1}<a_{M+1}$. But $a_{i}<a_{M+1}$ implies that $a_{i}$ is among the $M$ successful bids.

We can now prove

Theorem 4.1 If seller $i$ submits any bid $B_{i}$, then we necessarily have $a_{i} \leq B_{i} \leq$ $c_{i}$.

PROOF. To prove this theorem, we compare seller $i$ 's payoff when setting some $B_{i}<a_{i}$, with her payoff when only setting the same ask $a_{i}$, without submitting a $B_{i}<a_{i}$. We will show that using the second strategy strictly dominates using the first one. That implies that submitting a bid $B_{i}$ can only be part of an equilibrium

9 The weak inequality $a_{z} \leq a_{z+1}$ in this series implies that $i$ has the option to set an ask price equal to zero (in which case a total of $z+1$ sellers set ask zero, thus $0=a_{1}=\ldots=a_{z+1}<a_{z+2} \ldots$ ) or a strictly positive ask (and we have $a_{z}<a_{z+1}$ ). 
strategy when $B_{i} \geq a_{i}$. It is then easy to see that, necessarily, we have $B_{i} \leq c_{i}$ as well.

First suppose seller $i$ only submits some ask price $a_{i}$. Then the marginal bidder is $m$, as defined in (1). As was the case in the previous section, $r$ is the rank of ask $a_{i}$. If the ask is successful, it yields price $b_{r}$, which is the bid matched to seller $i$ 's ask. The payoff to seller $i$ equals that given in (2): ${ }^{10}$

$$
\pi_{i}=\left\{\begin{array}{cc}
b_{r}-c_{i} & \text { if } r \leq m \\
0 & \text { if } r>m
\end{array} .\right.
$$

We now derive seller $i$ 's payoff when she submits an ask price $a_{i}$ and a bid $B_{i}$ with $a_{i}>B_{i}$. In that case the marginal bidder is $M$, as defined in (4). When submitting a bid $B_{i}<a_{i}$, several things can now happen.

1. When the bid $B_{i}$ is successful, we have $B_{i} \geq \beta_{M}$. From lemma 4.1, $a_{i}$ cannot be successful, thus $r>M$, which implies $r>m$, since we either have $M=m$ or $M=m+1 .{ }^{11}$ Hence, the seller buys an additional allowance which she values at zero. The payoff of seller $i$ then equals $-B_{i}$.

2. If bid $B_{i}$ is not successful, $B_{i}<\beta_{M}$. Note that the number of successful bidders when $B_{i}$ is not taken into account, is equal to the number of successful bidders when $B_{i}$ is taken into account. Thus $M=m$. We have two subcases

(a) First suppose $a_{i}$ is successful, hence $r \leq M$, and thus $r \leq m$. Since the seller's own bid $B_{i}$ is not sucessful, ask $a_{i}$ is still matched with $b_{r}$. Only an allowance is sold and none is bought, which implies a payoff of $b_{r}-c_{i}$.

(b) Next, suppose that $a_{i}$ is not successful. In that case, an allowance is neither bought nor sold, and the payoff is 0 .

Combining the cases described above we find

$$
\pi_{i}=\left\{\begin{array}{cl}
b_{r}-c_{i} & \text { if } r \leq m \\
-B_{i} & \text { if } r>m \text { and } B_{i} \geq \beta_{M} \\
0 & \text { otherwise }
\end{array}\right.
$$

10 Since in the case considered here $a_{i}>B_{i} \geq 0$, we do not have to take the possibility of ties into account.

11 This can be seen as follows. The value $m$ denotes the marginal bidder when bid $B_{i}$ is not taken into account, whereas $M$ is the marginal bidder when $B_{i}$ is taken into account. Introducing an extra bidder either leaves the total amount of successful bidders unchanged, or it increases the number of successful bidders by 1 . Therefore, either $M=m$ or $M=m+1$. 
Comparing (2) and (5), we have that submitting only an ask price always weakly dominates, and is strictly better if it turns out that $B_{i} \geq \beta_{M}$. Thus, rather than setting a bid $B_{i}<a_{i}$, a seller is always better off only submitting the ask $a_{i}$. Hence, in equilibrium we never have $B_{i}<a_{i}$, which proves the first inequality in the theorem.

The inequality $B_{i} \leq c_{i}$ is obvious. Since a seller values an allowance at most at $c_{i}$, it never makes sense to submit a bid higher than this value, thus $B_{i} \leq c_{i}$. Combined with the previous result, we thus have $a_{i} \leq B_{i} \leq c_{i}$, if a bid $B_{i}$ is submitted.

We now have

Theorem 4.2 If seller $i$ submits any bid $B_{i}$, then it is a dominant strategy to set $a_{i}=0$.

PROOF. We will show that when seller $i$ submits a bid $B_{i}$, then setting $a_{i}=0$ yields a higher payoff than setting any $a_{i}$ such that $0<a_{i} \leq B_{i}$. From theorem 4.1, we know that we can disregard the option $a_{i}>B_{i}$.

We define a ranking $\hat{\beta}_{1} \geq \hat{\beta}_{2} \ldots$ as follows

$$
\hat{\beta}_{j} \equiv\left\{\begin{array}{cl}
\frac{1}{z} \sum_{k=1}^{z} \beta_{k} & \text { if } a_{i}>0 \text { and } j \leq z \\
\frac{1}{z+1} \sum_{k=1}^{z+1} \beta_{k} & \text { if } a_{i}=0 \text { and } j \leq z+1 \\
\beta_{j} & \text { otherwise }
\end{array}\right.
$$

Thus, $\hat{\beta}_{j}$ reflects the expected value of the bid matched to the $j$-th lowest (and possibly tied) ask $a_{j}$.

We have several cases to consider.

1. First assume the bid $B_{i}$ is successful, i.e. $B_{i} \geq \beta_{M}$. Submitting $a_{i}=0$ yields $\hat{\beta}_{1}-B_{i}$, and submitting $a_{i}>0$ yields $-B_{i}$ if $a_{i}$ is not successful, and at most $\beta_{z+1}-B_{i}$ if $a_{i}$ is successful. Since $\beta_{z+1}<\hat{\beta}_{1}$, it is a dominant strategy to set $a_{i}=0$.

2. Second assume that $B_{i}$ is not successful, hence $B_{i}<\beta_{M}$. Submitting $a_{i}=0$ yields $\hat{\beta}_{1}-c_{i}$. Any other ask $a_{i} \leq B_{i}$ will be successful, because of lemma 4.2, and yields a payoff of at most $\beta_{z+1}-c_{i}$. Again, it is a dominant strategy to set $a_{i}=0$.

Theorem 4.3 If seller $i$ sets $a_{i}=0$, then she will always set some $B_{i}>0$. 
Proof. We now define $\hat{b}_{j}=\frac{1}{z+1} \sum_{k=1}^{z+1} b_{k}$ for $j \leq z+1$ and $\hat{b}_{j}=b_{j}$ for $j>$ $z+1$. Again, $M$ is the marginal bidder when the bid of seller $i$ is also taken into account, as defined by (4). First note that only setting $a_{i}=0$ always yields a payoff of $\pi_{i}=\hat{b}_{1}-c_{i}$. Now consider what happens if seller $i$ sets a bid $B_{i}$ as well. There are three possibilities to consider.

1. Suppose $B_{i}<\beta_{M}$. In that case, seller $i$ 's bid is simply not successful and we have a payoff of $b_{1}-c_{i}$.

2. Consider the case in which $B_{i} \geq \beta_{M}$, but $B_{i}<b_{z+1}$. Here, bid $B_{i}$ is successful, but it is not among the $z+1$ highest bids that are matched to sellers with an ask price of 0 . Seller $i$ 's payoff now equals $\hat{b}_{1}-B_{i}$.

3. Finally, $B_{i}>b_{z+1}$. Again, $B_{i}$ is successful. But now, it also changes the expected revenue of setting ask price zero. Note that this expected revenue equals the average value of the $z+1$ highest bids. Compared to the case in which seller $i$ did not bid, $b_{z+1}$ now drops out of the $z+1$ highest bids, and is replaced by $B_{i}$. Hence, the expected payoff now equals $\hat{b}_{1}+\left(B_{i}-b_{z+1}\right) /(z+$ 1) $-B_{i}$.

Summarizing, we have

$$
\pi_{i}=\left\{\begin{array}{cll}
\hat{b}_{1}-c_{i} & \text { if } \quad B_{i}<\beta_{M} \\
\hat{b}_{1}-B_{i} & \text { if } \quad \beta_{M} \leq B_{i}<b_{z+1} \\
\hat{b}_{1}+\frac{B_{i}-b_{z+1}}{z+1}-B_{i} & \text { if } & B_{i}>b_{z+1}
\end{array}\right.
$$

With only $a_{i}=0$, we had an expected payoff of $\hat{b}_{1}-c_{i}$. Therefore, any bid $B_{i}<c_{i}$ improves upon this strategy. In the first case, both strategies yield the same payoff. In the second case, submitting a bid yields a higher payoff since $B_{i}<c_{i}$. In the third case, since $B_{i}-b_{z+1}>0$, expected payoff is higher as well.

We have now prove the following result:

Theorem 4.4 In equilibrium, every seller $i$ either sets an ask $a_{i}=0$ and $a$ bid price $B_{i} \in\left(0, c_{i}\right]$, or only an ask $a_{i}>0$.

PROOF. Given all the theorems proven above, the only thing left to prove is that any seller $i$ prefers to do any of the two options described in this theorem, rather than refrain from participating in the auction altogether. Not participating yields 0. Setting an ask $a_{i}=c_{i}$ never yields a negative payoff (since a seller only sells only when the bid $b_{r}$ to which she is matched has $b_{r} \geq a_{i}$ ), whereas there is a positive probability of 
making a strictly positive profit. Thus, any seller $i$ prefers to set an ask $c_{i}$ rather than dropping out of the auction.

In section 3, we showed that a seller can always improve upon the equilibrium described in Cason by submitting an ask $a_{i}=0$.This does not imply, however, that in the Nash equilibrium every seller will actually set an ask $a_{i}=0$. When other sellers are also following this strategy, setting $a_{i}=0$ no longer implies that seller $i$ is matched with the highest bid $b_{1}$. When $z$ other sellers are submitting an ask equal to zero as well, the expected value of the bid to which seller $i$ 's ask is matched equals the average value of the $z+1$ highest bids. This expected value can be lower than seller $i$ 's own valuation $c_{i}$ and/or her own bid $B_{i}$. Setting $B_{i}=c_{i}$ no longer guarantees a nonnegative pay-off, as was the case in the proof of theorem 3.1.

\section{Deriving the Equilibrium}

In this section, we give a tentative derivation of the appropriate equilibrium in the auction considered. It is beyond the scope of this paper to give a full derivation. A general solution will be very hard to find, given the fact that we have an asymmetric auction with a stochastic number of participants, both of which are notoriously hard to solve. Therefore, we make some highly simplifying assumptions, to give a taste for the proper analysis.

First, we consider the case in which all sellers set an ask price equal to zero. The analysis is then almost identical to a normal auction, with $n \equiv Q+N$ bidders; $Q$ who do not own an allowance, and try to buy it, and $N$ who do own an allowance, but have offered it at ask price zero. For simplicity, we also assume that the effect of the own bid on the expected price for which the own license is sold, is not taken into account by the sellers.

In general, ${ }^{12}$ in an auction with $n$ bidders, whose valuation follows some cumulative probability density function $F(\cdot)$, defined on some interval $[\underline{v}, \bar{v}]$, the expected utility for bidder $i$ of submitting a bid $b_{i}$, given that his valuation is $v_{i}$, equals

$$
U_{i}\left(b_{i}\left(v_{i}\right), v_{i}\right)=\rho\left(b_{i}\left(v_{i}\right)\right)\left(v_{i}-b_{i}\left(v_{i}\right)\right),
$$

with $\rho\left(b_{i}\left(v_{i}\right)\right)$ the endogenously determined probability that bid $b_{i}$ is successful. Note that

$$
\frac{d U_{i}}{d v_{i}}=\frac{\partial U_{i}}{\partial v_{i}}+\frac{\partial U_{i}}{\partial b_{i}} \frac{d b_{i}}{d v_{i}}
$$

12 The discussion here closely follows Wolfstetter (1996). 
But, when expected utility is maximized, we have, by definition $\partial U_{i} / \partial b_{i}=0$, hence, using (8),

$$
\frac{d U_{i}}{d v_{i}}=\rho\left(b_{i}\left(v_{i}\right)\right)
$$

In equilibrium, all bidders use the same bidding strategy $b^{*}\left(v_{i}\right)$. It can be shown that this bidding strategy is strictly increasing in $v_{i}$. The fact that the goods are awarded to the highest bidders therefore implies that, in equilibrium, they are awarded to the highest $v_{i}$ 's, and we can write

$$
\frac{d U_{i}}{d v_{i}}=\rho\left(v_{i}\right)
$$

Integrating yields

$$
U^{*}\left(v_{i}\right)=\int_{\underline{v}}^{v_{i}} \rho(x) d x .
$$

Since (8) now implies

$$
U^{*}\left(v_{i}\right)=\rho\left(v_{i}\right)\left(v_{i}-b^{*}\left(v_{i}\right)\right),
$$

thus the equilibrium bid strategy is given by

$$
b^{*}\left(v_{i}\right)=v_{i}-\frac{\int_{\underline{v}}^{v_{i}} \rho(x) d x}{\rho\left(v_{i}\right)} .
$$

With a simple single auction, in which only one unit of the good is sold, it is easy to see that $\rho\left(v_{i}\right)=F\left(v_{i}\right)^{n-1}$, and we get the well-known result ${ }^{13}$

$$
b^{*}\left(v_{i}\right)=v_{i}-\frac{\int_{\underline{v}}^{v_{i}} F(x)^{n-1} d x}{F\left(v_{i}\right)^{n-1}},
$$

which implies that the bid of bidder $i$ is equal to the expected value of the secondhighest bid, conditional on bidder $i$ 's bid being the highest. In an auction in which the goods are awarded to the $N$ highest bidders, who all pay their own bid, we have that the probability of winning equals the probability of $v_{i}$ being among the $N$ highest of $n$ independent draws of a random variable with density function $F(\cdot)$. This equals the

13 See e.g. McAfee and McMillan (1987) 
probability that $v_{i}$ is higher than the $(n-N)$ th order statistic of $n-1$ independent draws: ${ }^{14}$

$$
\rho\left(v_{i}\right)=\sum_{j=n-N}^{n-1} \frac{(n-1) !}{j !(n-1-j) !}\left[F\left(v_{i}\right)\right]^{j}\left[1-F\left(v_{i}\right)\right]^{n-1-j},
$$

hence

$$
b^{*}\left(v_{i}\right)=v_{i}-\frac{\int_{0}^{v_{i}}\left(\sum_{j=n-N}^{n-1} \frac{(n-1) !}{j !(n-1-j) !} F(x)^{j}[1-F(x)]^{n-1-j}\right) d x}{\left(\sum_{j=n-N}^{n-1} \frac{(n-1) !}{j !(n-1-j) !} F\left(v_{i}\right)^{j}\left(1-F\left(v_{i}\right)\right)^{n-1-j}\right)}
$$

Thus the bid of bidder $i$ equals the expected value of the $(N+1)$ st highest bid, conditional on his own valuation $v_{i}$ being the $N$-th highest.

To determine the expected payoff to a seller with valuation $v_{i}$ of submitting the bid $b^{*}\left(v_{i}\right)$ and setting the ask $a_{i}=0$, we proceed as follows. Note that the expected payoff of setting $a_{i}=0$ equals the average value of the $N$ highest bids minus the own valuation $v_{i}$. The expected payoff of following this strategy thus equals

$$
U^{*}\left(v_{i}\right)=\rho\left(v_{i}\right)\left(v_{i}-b^{*}\left(v_{i}\right)\right)+\left(\bar{b}_{N}^{*}-v_{i}\right)
$$

with $\rho\left(v_{i}\right)$ as defined in $(16), b^{*}\left(v_{i}\right)$ as defined in (17), and $\bar{b}_{N}^{*}$ the expected average of the $N$ highest bids. Define $B(b)$ as the probability density function of the equilibrium bids (thus $B(b)=P\left(b \geq b^{*}\left(v_{i}\right)\right)$, given the distribution of $v_{i}$, which is $F\left(v_{i}\right)$ ). The value $\bar{b}_{N}^{*}$ is then the expected value of the average of the $N$ highest order statistics of $n$ drawings from the probability distribution $B(b)$.

So far, however, we have assumed that all sellers set an ask price zero and a positive bid price. This is not necessarily the best thing to do. Suppose all the others are following such a strategy. The payoff of doing so as well then equals (18). Only submitting a strictly positive ask $a_{i}$, however, then yields the $N$ th highest bid for sure. Denote the expected value of the $N$ th highest bid as $E\left(b_{N}\right)$. The expected payoff of this alternative is

$$
U^{*}\left(a_{i}\right)=\left(E\left(b_{N}\right)-v_{i}\right) \cdot P\left(b_{N}>a_{i}\right) .
$$

Since the total number of bids now equals $n-1$, we have that $b_{N}$ is the $(n-N)$ th order statistic, hence

$$
F_{b_{N}}(b)=\sum_{j=n-N}^{n-1} \frac{(n-1) !}{j !(n-1-j) !}[B(b)]^{j}[1-B(b)]^{n-1-j},
$$

14 For more on order statistics see e.g. Mood, Graybill and Boes (1974). 
and

$$
U^{*}\left(a_{i}\right)=\left(E\left(b_{N}\right)-v_{i}\right) \cdot\left(1-F_{b_{N}}\left(a_{i}\right)\right),
$$

which can be used to find the optimal $a_{i}$ to set. ${ }^{15}$ The best possible strategy is found by comparing the payoffs in (18) and (21). Yet, any seller also has to take into account that the other sellers face the same problem, and that when they decide to set a positive ask rather than a positive bid and an ask price zero, the decision problem they face also changes. A full description of the equilibrium thus implies

1. A specification of the probability density function of the bidders' valuations, $\Phi(\cdot)$ and sellers' costs $H(\cdot)$.

2. An endogenously derived equilibrium set $S$, which is a subset of the support of $H(\cdot)$, that specifies which sellers will not submit an ask $a_{i}=0$ and bid $B_{i}>0$, but rather only an ask $a_{i}>0$.

3. Given the set $S$, the expected number of de facto bidders and sellers in the auction, plus the proper (truncated) probability densities $H^{\prime}(\cdot)$ and $H^{\prime \prime}(\cdot)$ for sellers who, respectively, offer an allowance at price zero plus a positive bid, and those that only offer a positive ask.

4. Given the information in 3, equilibrium bid and ask strategies for the three groups of agents.

It seems virtually impossible to find an analytical solution for this ${ }^{16}$. Also, it is not guaranteed that an equilibrium in pure strategies does exist.

\section{Conclusion}

Cason (1993) argued that the auction the EPA used to start the market for sulfur allowances, is not efficient. The set-up of the auction gives both buyers and sellers an incentive to understate their valuation of an allowance. Therefore, equilibrium prices are too low, and the market is not efficient. In this paper, we showed that the incentives

15 When seller $i$ is the only one setting an ask $a_{i}>0$, which is the case considered in the text, then it is easy to see that it is optimal to set $a_{i}=c_{i}$. Setting a lower $a_{i}$ implies that this seller also has to sell when $a_{i}<b_{N}<c_{i}$, which causes a loss. Setting a higher $a_{i}$ implies that when $c_{i}<b_{N}<a_{i}$, she will not sell, although it would have been profitable to do so. However, when other sellers also decide to set a strictly positive ask price, this argument no longer holds, and the optimal $a_{i}$ has to be derived along the lines described in the main text.

16 In fact, it even turned out to be impossible to derive an equilibrium for the simplest numerical example conceivable. 
in this particular auction are even more perverse than Cason suggested. In particular, we showed that sellers have an incentive to set their ask price equal to zero, while simultaneously hedging their bets by submitting a positive bid at the same time. We also showed that the proper equilibrium in this auction, when this possibility is taken into account, is impossible to find. What we do show is that, if an equilibrium exists, sellers either set only a positive ask price, or an ask price equal to zero, and a positive bid as well.

As far as we know, no one has ever simultaneously submitted a bid and an ask at the EPA auction. ${ }^{17}$ Nor has this strategy ever been suggested in the literature about the EPA auction. Also, other literature on auctions does not study the possibility. The latter is probably due to the fact that only in a peculiar setup like that of the EPA auction, it is profitable to follow such a strategy.

We now know that the EPA auction was a failure, in the sense that hardly any private parties sold allowances at the auctions. As a result, it took several years before an efficient market in sulfur allowances developed. Cason (1993) suggested that the main reason for this was that, given equilibrium strategies for buyers and sellers, equilibrium prices are too low, making the market inefficient. Our analysis shows that in the true equilibrium, the incentives for sellers in the auction are even more perverse, shedding even more doubt on the efficiency of such an auction. Therefore, it does not come as a surprise that potential sellers preferred to bypass the auctions altogether, and waited for an efficient market to develop.

\section{References}

CAson, T. N. (1993): "Seller Incentive Properties of EPA's Emission Trading Auction," Journal of Environmental Economics and Management, 25(2), 177-195. (1995): "An Experimental Investigation of the Seller Incentives in the EPA's Emission Trading Auction," American Economic Review, 85(4), 905-922.

Cason, T. N., And C. R. Plott (1996): "EPA's New Emissions Trading Mechanism: A Laboratory Evaluation," Journal of Environmental Economics and Management, 30(2), 133-160.

Dales, J. H. (1968): Pollution, Property, and Prices. University Press, Toronto.

Ellerman, D. A., R. Schmalensee, P. L. Joskow, J. P. Montero, and E. M. BAILEY (1997): "Emissions trading under the US Acid Rain Program, evaluation of compliance costs and allowance market performance," Center for Energy

17 The EPA does not release the identity of those who offered allowances for sale at the auction. 
and Environmental Policy Research, Massachusetts Institute of Technology, Cambridge, Massachusetts.

EPA ACID RAIN WEBSITE (1998): http://www.epa.gov/docs/acidrain/ardhome.html. Joskow, P. L., R. Schmalensee, And E. M. BAiley (1998): "The market for sulfur dioxide emissions," American Economic Review, 58, 669-685.

LiRofF, R. A. (1986): Reforming Air Pollution Regulation: The Toil and Trouble of EPA's Bubble. The Conservation Foundation, Washington, D.C.

McAfee, R. P., AND J. McMillan (1987): "Auctions and Bidding," Journal of Economic Literature, 25(2), 699-738.

Mood, A. M., F. A. Gray BILL, AND D. C. Boes (1974): Introduction to the theory of statistics, 3rd ed. McGraw Hill.

Schmalensee, R., P. L. Joskow, A. D. Ellerman, J. P. Montero, and E. M. BAILEY (1998): "An interim evaluation of sulfur dioxide emissions trading," Journal of Economic Perspectives, 12(3), 53-68.

STAVINS, R. N. (1998): "What can we learn from the grand policy experiment? Lessons from SO2 allowance trading," Journal of Economic Perspectives, 12(3), 69-88.

Vickrey, W. (1961): "Counterspeculation, Auctions, and Competitive Sealed Tenders," Journal of Finance, 16, 8-37.

Wolfstetter, E. (1996): "Auctions: An Introduction," Journal of Economic Surveys, 10(4), 367-420. 


\begin{tabular}{|c|c|c|c|c|c|c|}
\hline $\begin{array}{l}\text { Year of } \\
\text { auction }\end{array}$ & $\begin{array}{l}\text { Vintage } \\
\text { year }^{a}\end{array}$ & $\begin{array}{l}\text { Offered } \\
\text { by EPA }\end{array}$ & $\begin{array}{l}\text { Privately } \\
\text { offered }\end{array}$ & Bid for & Sold & $\begin{array}{l}\text { Private } \\
\text { trades }^{b}\end{array}$ \\
\hline 1993 & 1995 & 50,000 & 95,010 & 321,354 & 50,010 & \multirow{2}{*}{$\begin{array}{l}\text { Not } \\
\text { available }\end{array}$} \\
\hline 1993 & 2000 & 100,000 & 30,500 & 283,506 & 100,000 & \\
\hline 1994 & 1995 & 50,000 & 58,001 & 294,354 & 50,000 & \multirow[t]{3}{*}{$900,000^{c}$} \\
\hline 1994 & 2000 & 25,000 & 50,000 & 110,489 & 25,400 & \\
\hline 1994 & 2001 & 100,000 & 47,000 & 489,399 & 100,800 & \\
\hline 1995 & 1995 & 150,000 & 8,306 & 255,371 & 50,600 & \multirow[t]{3}{*}{$1,900,000$} \\
\hline 1995 & 2001 & 25,000 & 7,000 & 70,286 & 25,400 & \\
\hline 1995 & 2002 & 100,000 & 7,000 & 236,928 & 100,400 & \\
\hline 1996 & 1996 & 150,000 & 8,000 & 911,735 & 150,000 & \multirow[t]{3}{*}{$4,400,000$} \\
\hline 1996 & 2002 & 25,000 & 7,000 & 148,026 & 25,000 & \\
\hline 1996 & 2003 & 100,000 & 7,000 & 404,634 & 100,000 & \\
\hline 1997 & 1997 & 150,000 & 0 & $1,224,582$ & 150,000 & \multirow[t]{3}{*}{$7,900,000$} \\
\hline 1997 & 2003 & 25,000 & 0 & 128,543 & 25,000 & \\
\hline 1997 & 2004 & 100,000 & 0 & 553,406 & 100,000 & \\
\hline 1998 & 1998 & 150,000 & 0 & 767,097 & 150,000 & \multirow{2}{*}{$\begin{array}{l}\text { Not } \\
\text { Available }\end{array}$} \\
\hline 1998 & 2005 & 125,000 & 0 & 509,009 & 125,000 & \\
\hline
\end{tabular}

${ }^{a}$ year from which the auctioned allowances can be used.

${ }^{b}$ trade between economically distinct organizations in a given year.

${ }^{c}$ April to December.

Source: EPA website

Table 1: The market for $\mathrm{SO}_{2}$ allowances. 